Tom 26 № 1

УДК 519.216.3

\title{
ОЦЕНИВАНИЕ СОСТОЯНИЙ СТОХАСТИЧЕСКИХ МНОГОШАГОВЫХ ВКЛЮЧЕНИЙ
}

\section{Б. И. Ананьев}

Рассмотрены многошаговые стохастические включения вида $z_{k} \in H_{k}\left(z_{k-1}, \omega\right)$, где $z_{k} \in Z_{k}=X_{k} Y_{k}$, $k \in 1: N$. Проекция $z_{k}$ на $X_{k}$ считается ненаблюдаемым, а проекция на $Y_{k}$ - наблюдаемым состоянием. Элемент $\omega$ принадлежит вероятностному пространству $(\Omega, \mathcal{F}, P)$, а мультиотображение $H_{k}(z, \cdot)$ является измеримым относительно $\sigma$-алгебры $\mathcal{G}_{k}$. Последние $\sigma$-алгебры полагаются независимыми при разных $k$, а их объединение $\mathcal{F}_{k}=\sigma\left(\bigcup_{i \in 1: k} \mathcal{G}_{i}\right) \subset \mathcal{F}$ характеризует возрастающее накопление информации. Исследуются три способа оценивания ненаблюдаемых состояний, которые основаны на разных подходах к формированию множества переходных вероятностей. Показано, что эти способы приводят к различным множествам условных распределений для ненаблюдаемых состояний процесса. Частично изучен вопрос о достаточных условиях совпадения рассмотренных схем фильтрации и доказано, что для конечных фазовых пространств эти схемы совпадают в случае неатомического вероятностного пространства. Введен новый класс лебеговских селекторов для произвольных мультиотображений и установлено, что он не пуст, в частности, для измеримых простых прямоугольников на неатомическом пространстве. Доказано, что в лебеговском классе для простых включений и селекторов, заданных на неатомическом вероятностном пространстве, схемы фильтрации также совпадают.

Ключевые слова: оценивание, фильтрация, стохастические включения, селекторы, переходные вероятности, условные распределения.

\section{B. I. Ananyev. Estimation of states of multistage stochastic inclusions.}

Multistage stochastic inclusions of the form $z_{k} \in H_{k}\left(z_{k-1}, \omega\right)$, where $z_{k} \in Z_{k}=X_{k} Y_{k}$ and $k \in 1: N$, are considered. We regard the projection of $z_{k}$ to $X_{k}$ as an unobservable state and the projection of $z_{k}$ to $Y_{k}$ as an observable state. The element $\omega$ belongs to a probability space $(\Omega, \mathcal{F}, P)$, and the multifunction $H_{k}(z, \cdot)$ is measurable with respect to a $\sigma$-algebra $\mathcal{G}_{k}$. These $\sigma$-algebras are supposed to be independent for different $k$, and their union $\mathcal{F}_{k}=\sigma\left(\bigcup_{i \in 1: k} \mathcal{G}_{i}\right) \subset \mathcal{F}$ characterizes an increasing accumulation of information. We consider three ways of estimating the unobservable states based on different methods of forming the set of transition probabilities. It is shown that these ways result in different sets of conditional distributions for the unobservable states of the process. The question of sufficient conditions for the coincidence of the considered filtering schemes is partially studied, and it is proved that, for finite state spaces, these schemes coincide in the case of a nonatomic probability space. A new class of Lebesgue selections is introduced for arbitrary multifunctions and is shown to be nonempty, in particular, for measurable simple rectangles on a nonatomic space. It is proved that the filtering schemes also coincide in the Lebesgue class for simple inclusions and selections defined on a nonatomic probability space.

Keywords: estimation, filtering, stochastic inclusions, selections, transition probabilities, conditional distributions.

MSC: 93E10, 62L12, 34G25

DOI: $10.21538 / 0134-4889-2020-26-1-12-26$

\section{1. Введение}

Данная статья продолжает исследования [1]. Результаты настоящей работы могут использоваться в задачах восстановления входных воздействий и задачах коррекции движения механических систем при наличии коммуникационных ограничений в виде неточных цифровых каналов связи $[2 ; 3]$. Другие возможные подходы к оцениванию в статистически неопределенных ситуациях были предложены, например, в публикациях [4-6], где содержится обширная библиография по теме. Вместе с тем за последние 10-15 лет тематика статистическинеопределенного оценивания постоянно развивалась в разных направлениях. Так, например, 
в книге [7] представлены задачи оценивания, решаемые с использованием интервального анализа и теории размытых множеств. В статье [8] доказано, что множества достижимости стохастических дифференциальных включений являются подмножествами некоторых многозначных стохастических уравнений. В монографии [9] содержатся многочисленные примеры задач нелинейной фильтрации и их инженерные применения. Новые возможности для исследования задач стохастически-неопределенного оценивания открываются при использовании подхода из [10], где случайные множества рассматриваются как результат неточных наблюдений случайных переменных. Авторы изучают верхние и нижние вероятности, индуцированные случайными множествами, и дают оценку для вероятностных распределений. Проблема увязывается с существованием селекторов для многозначных отображений. Данный подход применяется в настоящей работе для задач статистически-неопределенного оценивания. Для решения абстрактной задачи фильтрации с заданными вероятностями перехода используется известная теория из монографии [11].

Введем ряд обозначений и понятий, принятых в статье. Измеримым пространством называется пара $(\Omega, \mathcal{F})$, состоящая из произвольного множества $\Omega$ и заданной на нем $\sigma$-алгебры $\mathcal{F}$. Если на пространстве задана счетно-аддитивная мера $P$, то тройка $(\Omega, \mathcal{F}, P)$ определяется как вероятностное пространство. Борелевская $\sigma$-алгебра на топологическом пространстве $X$ обозначается символом $\mathbb{B}_{X}$. Если $X$ - метризуемое пространство, то под символом $\mathbb{P}(X)$ понимается множество всех вероятностных мер на измеримом пространстве $\left(X, \mathbb{B}_{X}\right)$. В задачах оценивания и стохастического управления рассматриваемые метризуемые пространства часто должны обладать дополнительными свойствами. А именно вводятся борелевские пространства. Пространство $X$ называется борелевским, если оно гомеоморфно борелевскому подмножеству полного сепарабельного метрического пространства (польского). Всякое несчетное борелевское пространство имеет мощность с (континуума). Более того, два борелевских пространства борелевски изоморфны тогда и только тогда, когда они обладают одинаковой мощностью, [11, предложение 7.16]. В частности, всякое борелевское пространство борелевски изоморфно $[0,1]$ с $\sigma$-алгеброй $\mathbb{B}_{[0,1]}$. Декартово произведение (счетное) множеств $X_{1}, X_{2}, \ldots$ обозначается через $X_{1} X_{2} \cdots$. Если в множествах $X_{1}, X_{2}, \ldots$ заданы $\sigma$-алгебры $\mathcal{F}_{X_{1}}, \mathcal{F}_{X_{2}}, \ldots$, то их произведением $\mathcal{F}_{X_{1}} \mathcal{F}_{X_{2}} \cdots$ в $X_{1} X_{2} \cdots$ является наименьшая $\sigma$-алгебра, содержащая все множества вида $A_{1} A_{2} \cdots$, где $A_{i} \in \mathcal{F}_{X_{i}}, i=1,2, \ldots$ В случае топологических пространств имеем $\mathbb{B}_{X_{1}} \mathbb{B}_{X_{2}} \cdots=\mathbb{B}_{X_{1} X_{2} \cdots}$, где в $X_{1} X_{2} \cdots$ введена топология произведения. Для произвольного семейства $\mathcal{P}$ подмножеств какого-либо множества $X$ порождаемая семейством $\sigma$-алгебра обозначается как $\sigma(\mathcal{P})$. Если $f: \Omega \rightarrow X$ - отображение из одного измеримого пространства $(\Omega, \mathcal{F})$ в другое $(X, \mathcal{A})$, то $f$ называется $\mathcal{F} \mid \mathcal{A}$-измеримым при условии, что $f^{-}(A)=\{\omega \mid f(\omega) \in A\} \in \mathcal{F}, \forall A \in \mathcal{A}$. Математическое ожидание обозначается символом Е. Для числовой $\mathcal{F} \mid \mathbb{B}_{\mathbb{R}}$-измеримой и интегрируемой $(\mathrm{E}|f|<\infty)$ функции $f$ на вероятностном пространстве $(\Omega, \mathcal{F}, P)$ условное математическое ожидание относительно $\sigma$-алгебры $\mathcal{B} \subset \mathcal{F}$ маркируется символом $\mathrm{E}(f \mid \mathcal{B})$. Для случайных функций в гильбертовом или банаховом пространстве $X$ также определяется условное среднее по формуле $h(\mathrm{E}(f \mid \mathcal{B}))=\mathrm{E}(h(f) \mid \mathcal{B}), \forall h \in X^{*}$, где $X^{*}$ - сопряженное пространство к $X$. В случае $\mathcal{B}=y^{-}(\mathcal{A})$, где $y: \Omega \rightarrow X$ есть $\mathcal{F} \mid \mathcal{A}$-измеримое отображение из $(\Omega, \mathcal{F})$ в $(X, \mathcal{A})$, часто пишут $\mathrm{E}(f \mid y)$ вместо $\mathrm{E}\left(f \mid y^{-}(\mathcal{A})\right)$.

При повторении случайного эксперимента некоторые случайные величины и отображения относят к "наблюдаемым", а другие к "ненаблюдаемым". Далее кавычки при упоминании состояний опускаются. Пусть имеется $\mathcal{F} \mid \mathbb{B}_{X}$-измеримое ненаблюдаемое отображение $x: \Omega \rightarrow X$ из вероятностного пространства $(\Omega, \mathcal{F}, P)$ в борелевское пространство $X$, а также $\mathcal{F} \mid \mathbb{B}_{Y}$-измеримое наблюдаемое отображение $y: \Omega \rightarrow Y$ из того же вероятностного пространства в другое борелевское пространство $Y$. Тогда по теореме [12, теорема II.7.5] существует регулярное условное распределение $q(A \mid y)=P(x \in A \mid y)=\mathrm{E}\left(I_{x^{-}(A)} \mid y\right)$ (равенство понимается почти наверное по мере $P y^{-}$при фиксированном $\left.A \in \mathbb{B}_{X}\right)$. Здесь $q(\cdot \mid y) \in \mathbb{P}(X)$ при фиксированном $y \in Y, I_{B}-$ характеристическая функция множества $B$. Такое распределение $q(A \mid y)$ называется стохастическим ядром на $X$ при условии $Y$ или переходной вероятностъю из $Y$ в $X$. Извест- 
но [11, следствие 7.25.1], что если $X$ - борелевское пространство, то $\mathbb{P}(X)$ также борелевское пространство, когда оно наделено слабой топологией. Каждое стохастическое ядро $q(\cdot \mid y)$ представляет собой семейство вероятностных мер из $\mathbb{P}(X)$, параметризованное посредством $y \in Y$. Борелевская измеримость ядер означает следующее. Пусть $\gamma: Y \rightarrow \mathbb{P}(X)$ - отображение вида $\gamma(y)=q(\cdot \mid y)$, тогда $\gamma^{-}\left(\mathbb{B}_{\mathbb{P}(X)}\right) \subset \mathbb{B}_{Y}$. Понятие борелевской измеримости ядер эквивалентно борелевской измеримости функций $q(A \mid y)$ по $y$ для всех $A \in \mathbb{B}_{X}$. Стохастическое ядро $q(\cdot \mid y)$ называется непрерывным, если отображение $\gamma$ непрерывно.

Пусть отображение $f: X \rightarrow H$ таково, что оно $\mathbb{B}_{X} \mid \mathbb{B}_{H}$-измеримо и конечен интеграл $\mathrm{E}\|f(x)\|$ для композиции $f(x)$ отображений $f$ и $x$, где $H$ - гильбертово пространство. Тогда с помощью стохастического ядра $q(\cdot \mid y)$ можно записать равенство для условного ожидания $\mathrm{E}(f(x) \mid y)=\int_{X} f(x) q(d x \mid y)$. Если же $\mathrm{E}\|f(x)\|^{2}<\infty$, то последний интеграл решает задачу наилучшего приближения отображения $f(x)$ с помощью наблюдаемых данных:

$$
\mathrm{E}\|f(x)-g(y)\|^{2} \rightarrow \min _{g(\cdot)}
$$

по всем $\mathbb{B}_{Y} \mid \mathbb{B}_{H}$-измеримым отображениям $g: Y \rightarrow H$ со свойством $\mathrm{E}\|g(y)\|^{2}<\infty$.

Нам потребуется следующая теорема о разложении стохастических ядер на произведении борелевских пространств.

Теорема 1 [11, предложение 7.27]. Пусть $(Z, \mathcal{B})$ - измеримое пространство, $X$ и $Y-$ борелевские пространства, и пусть $q(\cdot \mid z)$ - стохастическое ядро на XY при условии $Z$. Предположим, что функиия $q(B \mid z) \mathcal{B}$-измерима по z при любом множестве $B \in \mathbb{B}_{X Y}$. Тогда существуют стохастическое ядро $r(\cdot \mid z, y)$ на $X$ при условии $Z Y$ и стохастическое ядро $s(\cdot \mid z)$ на $Y$ при условии $Z$ такие, что $r(A \mid z, y) \mathcal{B B}_{Y}$-измеримо по $(z, y)$ для любых множеств $A \in \mathbb{B}_{X}, s(B \mid z) \mathcal{B}$-измеримо по z для любых множеств $B \in \mathbb{B}_{Y}, u$

$$
q(A B \mid z)=\int_{B} r(A \mid z, y) s(d y \mid z) \quad \forall A \in \mathbb{B}_{X}, \quad \forall B \in \mathbb{B}_{Y},
$$

причем $s(B \mid z)=q(X B \mid z)$.

Если $x: \Omega \rightarrow X$ и $y: \Omega \rightarrow Y-$ борелевски измеримые случайные отображения, то из приведенной теоремы, в частности, следует существование регулярной условной вероятности. Действительно, определим меру $q(A B)=P\left(x^{-}(A) \cap y^{-}(B)\right)$. Применяя теорему, получаем стохастическое ядро $r(A \mid y)$, которое и является регулярной условной вероятностью.

\section{2. Проблема многошаговой фильтрации}

В данном разделе рассмотрим задачу фильтрации с известными и неопределенными переходными вероятностями.

\section{1. Фильтрация с известными вероятностями перехода}

Пусть заданы борелевские пространства $X_{0}, \ldots, X_{N}$ (ненаблюдаемые состояния) и пространства $Y_{0}, \ldots, Y_{N}$ (наблюдаемые состояния). Число $N$ - конечный горизонт. Введем произведения пространств $Z_{0}=X_{0} Y_{0}, \ldots, Z_{N}=X_{N} Y_{N}$. Пусть также заданы вероятностное пространство $(\Omega, \mathcal{F}, P)$ и многошаговые уравнения

$$
z_{k}=h_{k}\left(z_{k-1}, \omega\right), \quad k \in 1: N
$$

осуществляющие случайный переход из $Z_{k-1}$ в $Z_{k}$. Отображения $h_{k}: Z_{k-1} \Omega \rightarrow Z_{k}$ предполагаются $\mathbb{B}_{Z_{k-1}} \mathcal{G}_{k} \mid \mathbb{B}_{Z_{k}}$-измеримыми, где возрастающий поток $\sigma$-алгебр $\mathcal{F}_{k}=\sigma\left(\bigcup_{i \in 1: k} \mathcal{G}_{i}\right) \subset \mathcal{F}$ 
характеризует накопление информации. Набор $\sigma$-алгебр $\mathcal{G}_{1}, \ldots, \mathcal{G}_{N}$ примем независимым в совокупности. Начальное состояние $z_{0}: \Omega \rightarrow Z_{0}$ является также случайным $\mathcal{F}_{0} \mid \mathbb{B}_{Z_{0} \text {-измеримым }}$ отображением, где $\mathcal{F}_{0}$ независимо от $\mathcal{F}_{N}$. Таким образом, основное вероятностное пространство предполагается достаточно "богатым". Последовательность $z_{k}$ образует марковский процесс. Введем стохастические ядра на $Z_{k}$ при условии $Z_{k-1}$ и распределение начального состояния:

$$
\begin{gathered}
q_{k}(D \mid z)=P\left(h_{k}^{-}(D)_{z}\right) \quad \forall D \in \mathbb{B}_{Z_{k}}, \forall z \in Z_{k-1}, \\
q_{0}(D)=P\left(z_{0}^{-}(D)\right) \forall D \in \mathbb{B}_{Z_{0}},
\end{gathered}
$$

где символом $U_{z}$ обозначено сечение множества $U \subset Z_{k-1} \Omega$ (в нашем случае $U=h_{k}^{-}(D)$ ). Стохастические ядра $q_{k}(\cdot \mid z)$ являются борелевскими. Данный факт вытекает из следующего, более общего, утверждения.

Лемма 1. Пусть $\left(\Omega_{i}, \mathcal{F}_{i}\right), i=1,2,-$ два измеримых пространства и $q(\cdot \mid x)$ - переходная вероятность из $\Omega_{1}$ в $\Omega_{2}$, такая что при каждом $x \in \Omega_{1}$ мера $q(\cdot \mid x)$ является вероятностной на измеримом пространстве $\left(\Omega_{2}, \mathcal{F}_{2}\right)$ и при каждом множестве $B \in \mathcal{F}_{2}$ функция $q(B \mid x)$ $\mathcal{F}_{1} \mid \mathbb{B}_{[0,1]}$-измерима. Тогда для всякого множества $D \in \mathcal{F}_{1} \mathcal{F}_{2}$, измеримого в $\sigma$-алгебре произведения, функция $\Lambda_{D}(x)=q\left(D_{x} \mid x\right)$ будет $\mathcal{F}_{1} \mid \mathbb{B}_{[0,1]}$-измеримой.

Д о к а з а т е л ь с т в о. Похожее утверждение содержится в [11, следствие 7.26.1], но там предполагается, что измеримые пространства - борелевские. Мы же устанавливаем более общий факт. Согласно [13, предложение III.1.2] выполняется включение $D_{x} \in \mathcal{F}_{2}$ для всякого $x \in \Omega_{1}$. Поэтому функция $\Lambda_{D}(x)$ корректно определена. Пусть $\mathcal{D}-$ семейство подмножеств из $\Omega_{1} \Omega_{2}$, для которых функция $\Lambda_{D}(x)$ измерима по $x$. Это семейство замкнуто относительно пересечений, поскольку $\Lambda_{D_{1} \cap D_{2}}(x)=\Lambda_{D_{1}}(x) \wedge \Lambda_{D_{2}}(x)$, где $a \wedge b=\min \{a, b\}$. Кроме того, $\Lambda_{D_{2} \backslash D_{1}}(x)=\Lambda_{D_{2}}(x)-\Lambda_{D_{1}}(x)$, если $D_{1} \subset D_{2}$. Если же $\cup_{i=1}^{\infty} D_{i}=D$ и $D_{i} \subset D_{i+1}$, то $\Lambda_{D_{i}}(x) \uparrow \Lambda_{D}(x)$. Таким образом, семейство $\mathcal{D}$ представляет собой систему Дынкина, устойчивую относительно пересечений. $\mathrm{K}$ тому же любой прямоугольник $D_{1} D_{2} \in \mathcal{D}$. По теореме $\left[12\right.$, теорема II.3.2] имеем $\mathcal{F}_{1} \mathcal{F}_{2} \subset \sigma(\mathcal{D}) \subset \mathcal{D}$.

Здесь и далее символ $\square$ означает окончание доказательства.

Используя начальное распределение, определим стохастическое ядро $r_{0}\left(\cdot \mid y_{0}\right)$ на $X_{0}$ при условии $Y_{0}$ для ненаблюдаемого состояния согласно теореме 1 и формуле (1.1):

$$
q_{0}(A B)=\int_{B} r_{0}(A \mid y) s_{0}(d y)
$$

где $s_{0}(B)=q_{0}\left(X_{0} B\right)$. Далее иногда полагается, что $s_{0}(B)=\delta_{y_{0}}(B)$, где $\delta_{y_{0}}-\delta$-функция Дирака. На первом шаге введем прогноз условного распределения на $Z_{1}$ за один шаг: $\bar{f}_{1}\left(D \mid r_{0}\right)=$ $\int_{X_{0}} q_{1}\left(D \mid x, y_{0}\right) r_{0}\left(d x \mid y_{0}\right)$. Теперь для этого прогноза, еще раз используя теорему, определим стохастическое ядро $r_{1}\left(\cdot \mid r_{0}, y_{1}\right)$ на $X_{1}$ при условии $Y_{0} Y_{1} \ni\left[y_{0} ; y_{1}\right]$ для ненаблюдаемых состояний из $X_{1}$, исходя из формулы $\bar{f}_{1}\left(A B \mid r_{0}\right)=\int_{B} r_{1}\left(A \mid r_{0}, y\right) s_{1}\left(d y \mid r_{0}\right)$, где $s_{1}\left(B \mid r_{0}\right)=\bar{f}_{1}\left(X_{1} B \mid r_{0}\right)$. Процесс фильтрации может быть продолжен. Пусть условное ядро $r_{k-1}\left(\cdot \mid r_{0}, y^{k-1}\right)$ на $X_{k-1}$ в ситуации, когда $Y_{0} Y_{1} \cdots Y_{k-1} \ni\left[y_{0} ; y^{k-1}\right]$, где символ $y^{i}$ означает упорядоченный набор $\left[y_{1} ; \ldots ; y^{i}\right]$, уже найдено. Тогда на $k$-м шаге вводим прогноз условного распределения на $Z_{k}$ за один шаг:

$$
\bar{f}_{k}\left(D \mid r_{k-1}\right)=\int_{X_{k-1}} q_{k}\left(D \mid x, y_{k-1}\right) r_{k-1}\left(d x \mid r_{0}, y^{k-1}\right), \quad k \in 2: N \text {. }
$$


Теперь для прогноза из $(2.4)$, используя теорему 1 , определим стохастическое ядро $r_{k}\left(\cdot \mid r_{0}, y^{k}\right)$ на $X_{k}$ при условии $Y_{0} Y_{1} \cdots Y_{k} \ni\left[y_{0} ; y^{k}\right]$ для ненаблюдаемых состояний из $X_{k}$, исходя из формулы

$$
\bar{f}_{k}\left(A B \mid r_{k-1}\right)=\int_{B} r_{k}\left(A \mid r_{0}, y^{k-1}, y\right) s_{k}\left(d y \mid r_{k-1}\right),
$$

где $s_{k}\left(B \mid r_{k-1}\right)=\bar{f}_{k}\left(X_{k} B \mid r_{k-1}\right)$.

Для отображений из (2.1) будем иметь равенство $P\left(h_{k}\left(z_{k-1}, \omega\right) \in A Y_{k} \mid y_{0}, y^{k}\right)=r_{k}\left(A \mid r_{0}, y^{k}\right)$ для $\forall A \in \mathbb{B}_{X_{k}}$ почти наверное по $\left[y_{0} ; y^{k}\right]$.

Рассмотрим также частный случай многошаговой фильтрации, где вместо уравнений (2.1) имеются уравнения

$$
z_{k}=h_{k}\left(x_{k-1}, \omega\right), \quad k \in 1: N,
$$

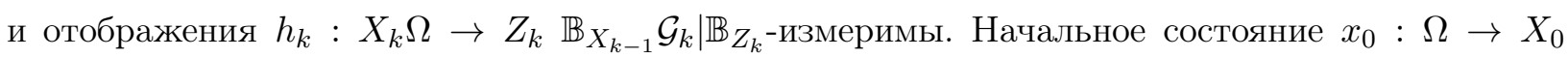
предполагается $\mathcal{F}_{0} \mid \mathbb{B}_{X_{0}}$-измеримым. Тогда начальным распределением служит мера $r_{0}(\cdot)$, не зависящая от $y_{0}$, а условные распределения $r_{k}\left(A \mid r_{0}, y^{k}\right)$ также не зависят от $y_{0}$. Отметим, что согласно [11, пр. 7.29] зависимость ядер $r_{k}$ от $r_{0}, y^{k}$ является борелевской, а также, что переходные вероятности (2.2) играют основную роль в процессе фильтрации и могут быть заданы априори без уравнений (2.1) или (2.6).

Рассмотрим случай абсолютно непрерывных мер. Пусть для простоты $X_{k}=\mathbb{R}^{n}, Y_{k}=\mathbb{R}^{m}$ и $q_{k}\left(D \mid z_{k-1}\right)=\int_{D} u_{k}\left(z \mid z_{k-1}\right) d z$, где $D \in \mathbb{B}_{\mathbb{R}^{n+m}}, 0 \leqslant u_{k}\left(z \mid z_{k-1}\right)$ - борелевские по переменным $z, z_{k-1}$ плотности вероятности при условии $z_{k-1}$. Пусть также $q_{0}(D)=\int_{D} u_{0}(z) d z$, где $u_{0}-$ начальная борелевская плотность вероятности. Тогда для условной меры $r_{0}$ из (2.3) плотность имеет вид $\hat{u}_{0}\left(x \mid y_{0}\right)=u_{0}\left(x, y_{0}\right) / \int_{\mathbb{R}^{n}} u_{0}\left(x, y_{0}\right) d x$, а плотность условного прогноза $(2.4)$ запишется KaK

$$
\bar{u}_{k}\left(z \mid y_{0}, y^{k-1}\right)=\int_{\mathbb{R}^{n}} u_{k}\left(z \mid x, y_{k-1}\right) \hat{u}_{k-1}\left(x \mid y_{0}, y^{k-1}\right) d x, \quad k \in 2: N .
$$

Наконец, плотность для условного ядра $r_{k}\left(\cdot \mid r_{0}, y^{k}\right)$ из $(2.5)$ будет иметь вид

$$
\hat{u}_{k}\left(x \mid y_{0}, y^{k}\right)=\bar{u}_{k}\left(x, y_{k} \mid y_{0}, y^{k-1}\right) / \int_{\mathbb{R}^{n}} \bar{u}_{k}\left(x, y_{k} \mid y_{0}, y^{k-1}\right) d x .
$$

В частном случае (2.6) в формулах $(2.7),(2.8)$ не будет зависимости от $y_{0}$.

\section{2. Фильтрация в статистически неопределенном случае}

Предположим, что случайный переход из $Z_{k-1}$ в $Z_{k}$ осуществляется согласно включению

$$
z_{k} \in H_{k}\left(z_{k-1}, \omega\right)
$$

что соответствует отсутствию некоторых статистических данных о распределении переходной вероятности. Здесь $H_{k}-$ многозначное отображсение (далее мультиотображение или мультифункиия). Множество всех подмножеств множества $X$ будет обозначаться через $\mathcal{P}(X)$. Пусть $H: X \rightarrow \mathcal{P}(Y)$ является произвольным мультиотображением. Верхний прообраз множества $B$ для $H$ определяется как $H^{*}(B)=\{x \in X \mid H(x) \cap B \neq \varnothing\}$. Нижний прообраз для $H$ задается формулой $H_{*}(B)=\{x \in X \mid H(x) \subset B\}$. Если $H$ зафиксировано, то прообразы будем записывать как $B^{*}$ и $B_{*}$ соответственно. Свойства прообразов перечислены в $[14$, леммы $1.2 .3,1.2 .4]$. Если на множествах $X, Y$ заданы $\sigma$-алгебры $\mathcal{A}, \mathcal{B}$ соответственно, то вводится 
понятие сильной измеримости $\left(\mathcal{A} \mid \mathcal{B}\right.$-измеримости), что означает $H^{*}(B) \in \mathcal{A}$ для всех $B \in \mathcal{B}$. Поскольку $\left(H_{*}\left(B^{c}\right)\right)^{c}=H^{*}(B)$, где $B^{c}=Y \backslash B$, то понятие сильной измеримости можно формулировать с помощью нижнего прообраза $H_{*}: \mathcal{B} \rightarrow \mathcal{A}$. Если на измеримом пространстве $(X, \mathcal{A})$ задана вероятностная мера $P$, то на измеримом пространстве $(Y, \mathcal{B})$ с помощью мультиотображения $H$ определяются функции множества $P^{*}(B)=P\left(B^{*}\right)$ и $P_{*}(B)=P\left(B_{*}\right)$. Эти функции множества (верхняя вероятность и нижняя вероятность), вообще говоря, не являются вероятностными мерами. Они сопряжсны в том смысле, что $P_{*}(B)=1-P^{*}\left(B^{c}\right)$ для всех $B \in \mathcal{B}$. Верхняя вероятность - полунепрерывная снизу и бесконечно альтернирующая [15]. В некоторых случаях будем писать $P_{H}^{*}$, чтобы подчеркнуть зависимость верхней вероятности от мультиотображения $H$.

Для $\mathcal{A} \mid \mathcal{B}$-измеримой мультифункции $H: X \rightarrow \mathcal{P}(Y)$ определяется множество измеримых селекторов:

$$
S(H)=\left\{h: X \rightarrow Y \mid \text { отображение } h-\mathcal{A} \mid \mathcal{B} \text { измеримо и } h(x) \in H(x) \forall x \in Y^{*}\right\} .
$$

На дополнении $X \backslash Y^{*}$ селектор задается произвольным постоянным значением из $Y$. Для всякого селектора $h \in S(H)$ символом $P_{h}$ обозначается его вероятностное распределение на $Y$, задаваемое как $P_{h}(B)=P\left(h^{-}(B)\right) \forall B \in \mathcal{B}$, где $h^{-}(B)=\{x \mid h(x) \in B\}$. Объединяя все такие распределения, получаем множество $P_{H}=\left\{P_{h} \mid h \in S(H)\right\} \subset \mathbb{P}(Y)$ и числовое множество $P_{H}(B)=\left\{P_{h}(B) \mid h \in S(H)\right\} \subset[0,1]$. При условии $P\left(Y^{*}\right)=1$, которое всегда предполагается в данной работе, будем иметь включение $h^{-}(B) \cap Y^{*} \subset H^{*}(B)$, откуда $P_{h}(B) \leqslant P^{*}(B) \forall B \in \mathcal{B}$. Но тогда в силу сопряженности имеем неравенство $P_{*}(B) \leqslant P_{h}(B)$. Следовательно, для всякого селектора $h \in S(H)$ при условии $P\left(Y^{*}\right)=1$ выполняется включение $P_{h}(B) \in\left[P_{*}(B), P^{*}(B)\right]$ $\forall B \in \mathcal{B}$. Вводятся множества

$$
\Delta_{H}=\left\{p \in \mathbb{P}(Y) \mid p(B) \in P_{H}(B) \forall B \in \mathcal{B}\right\}, \quad M_{H}=\left\{p \in \mathbb{P}(Y) \mid p(B) \leqslant P^{*}(B) \forall B \in \mathcal{B}\right\} .
$$

Ясно, что выполняются включения $P_{H} \subset \Delta_{H} \subset M_{H}$. Множество $M_{H}$ всегда выпукло и во многих случаях слабо компактно. Однако селекторов упомянутой мультифункции может не существовать. Достаточным условием существования служит теорема Куратовского [16, теорема 5.1], согласно которой для сепарабельного метрического пространства $Y$ с $\mathcal{B}=\mathbb{B}_{Y}$ и

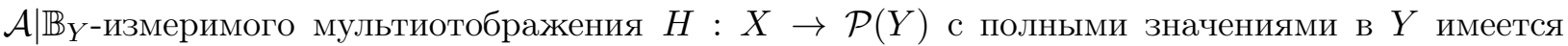
хотя бы один измеримый селектор. Далее предполагается, что во включениях (2.9) мультиотображения $H_{k}\left(z_{k-1}, \omega\right)$ являются $\mathbb{B}_{Z_{k-1}} \mathcal{G}_{k} \mid \mathbb{B}_{Z_{k}}$-измеримыми и $S\left(H_{k}\right) \neq \varnothing$ для всех $k \in 1: N$. Включения (2.9) формируют многозначный марковский процесс.

\section{3. Три схемы фильтрации для включений}

Для каждого $\mathbb{B}_{Z_{k-1}} \mathcal{G}_{k} \mid \mathbb{B}_{Z_{k}}$-измеримого селектора $h_{k}(z, \omega)$ из $S\left(H_{k}\right)$ определяем стохастическое ядро $q_{k}(\cdot \mid z)$ согласно $(2.2)$. Объединяя все такие ядра, введем множество

$$
\mathcal{Q}_{k}=\left\{q_{k}(\cdot \mid z) \text { из }(2.2) \mid h_{k} \in S\left(H_{k}\right)\right\}, \quad k \in 1: N .
$$

Далее на первом шаге вычисляем множество ядер прогноза:

$$
\bar{F}_{1}=\bigcup_{q_{1} \in \mathcal{Q}_{1}}\left\{\bar{f}_{1} \mid \bar{f}_{1}\left(\cdot \mid r_{0}\right)=\int_{X_{0}} q_{1}\left(\cdot \mid x, y_{0}\right) r_{0}\left(d x \mid y_{0}\right)\right\}
$$

и для каждого $\bar{f}_{1} \in \bar{F}_{1}$ по формуле $\bar{f}_{1}\left(A B \mid r_{0}\right)=\int_{B} r_{1}\left(A \mid r_{0}, y\right) s_{1}\left(d y \mid r_{0}\right)$ построим семейство условных распределений $\mathcal{R}_{1}=\bigcup_{\bar{f}_{1} \in \bar{F}_{1}}\left\{r_{1}\left(\cdot \mid r_{0}, y_{1}\right)\right\}$. Пусть множество условных распределений 
$\mathcal{R}_{k-1}=\left\{r_{k-1}\left(\cdot \mid r_{0}, y^{k-1}\right)\right\}, k \geqslant 2$, уже построено. Тогда можем найти множество ядер прогноза на шаге $k$ :

$$
\bar{F}_{k}=\bigcup_{q_{k} \in \mathcal{Q}_{k}, r_{k-1} \in \mathcal{R}_{k-1}}\left\{\bar{f}_{k}\left(\cdot \mid r_{k-1}\right) \text { из }(2.4)\right\} .
$$

Множество условных распределений на шаге $k$ выводим по формуле

$$
\mathcal{R}_{k}=\bigcup_{\bar{f}_{k} \in \bar{F}_{k}}\left\{r_{k}\left(\cdot \mid r_{0}, y^{k}\right) \text { из }(2.5)\right\} .
$$

Назовем схему построения условных распределений по формулам (3.1), (3.2), (3.3) селекторной схемой. Нетрудно видеть, что основную роль в этой процедуре играют семейства ядер $\mathcal{Q}_{k}$.

Сформируем переходные вероятности иными способами. Обозначим символом $\mathbf{P}_{k}$ набор всевозможных борелевских ядер $q\left(\cdot \mid z_{k-1}\right)$ на $Z_{k}$ при условии $Z_{k-1}$. Каждое ядро из (3.1) принадлежит $\mathbf{P}_{k}$. Введем числовое семейство $\mathcal{Q}_{k}^{z}(D)=\left\{q_{k}(D \mid z)\right.$ из $\left.(2.2) \mid h_{k} \in S\left(H_{k}\right)\right\}, k \in 1: N$. Теперь можем определить множества

$$
\mathcal{D}_{k}=\left\{q \in \mathbf{P}_{k} \mid q(D \mid z) \in \mathcal{Q}_{k}^{z}(D) \quad \forall D \in \mathbb{B}_{Z_{k}} \forall z \in Z_{k-1}\right\} .
$$

По построению ясно, что $\mathcal{Q}_{k} \subset \mathcal{D}_{k}$. Для определения еще одного множества переходных вероятностей рассмотрим верхний прообраз $H_{k}^{*}(D)=\left\{[z, \omega] \mid H_{k}(z, \omega) \cap D \neq \varnothing\right\}$ и введем верхнюю вероятность $P_{k}^{* z}(D)=P\left(H_{k}^{*}(D)_{z}\right)$ в зависимости от $z \in Z_{k-1}$. Введем также множество

$$
\mathcal{M}_{k}=\left\{q \in \mathbf{P}_{k} \mid q(D \mid z) \leqslant P_{k}^{* z}(D) \quad \forall D \in \mathbb{B}_{Z_{k}} \forall z \in Z_{k-1}\right\} .
$$

Введенные множества включаются друг в друга:

$$
\mathcal{Q}_{k} \subset \mathcal{D}_{k} \subset \mathcal{M}_{k} \quad \forall k \in 1: N .
$$

Далее сконструируем две рекуррентные процедуры построения условных распределений, подобные (3.2), (3.3), используя множества (3.4), (3.5) вместо (3.1). Обозначим данные множества условных средних символами $\mathcal{R}_{k}^{\mathcal{D}}$ и $\mathcal{R}_{k}^{\mathcal{M}}$ соответственно. Согласно включениям (3.6) получаем:

$$
\mathcal{R}_{k} \subset \mathcal{R}_{k}^{\mathcal{D}} \subset \mathcal{R}_{k}^{\mathcal{M}} \quad \forall k \in 1: N .
$$

Введем мультиотображение $H_{k}^{z}(\cdot)=H_{k}(z, \cdot)$ при фиксированном $z \in Z_{k-1}$. Наряду с множествами (3.4), (3.5) будем рассматривать множества, образующие включения

$$
P_{H_{k}^{z}} \subset \Delta_{H_{k}^{z}} \subset M_{H_{k}^{z}} \quad \forall k \in 1: N,
$$

для фиксированных $z \in Z_{k-1}$ согласно (2.10).

Рассмотрим

П р и м е $\mathrm{p} 1$. Задан одношаговый процесс с конечным фазовым пространством $Z_{0}=$ $Z_{1}=\{0,1,2,3\}^{2}$. Пусть начальное распределение сосредоточено в двух точках: $P\left(\left\{z_{0}=z_{01}\right\}\right)=$ $P\left(\left\{z_{0}=z_{02}\right\}\right)=1 / 2$, где $z_{01}=(1,1), z_{02}=(2,2)$. На первом шаге к начальному распределению независимо добавляется вероятностное пространство с равномерным распределением в двух точках $\{a, b\}$. Мультиотображения из (2.9) задаются соотношениями

$$
\begin{array}{ll}
H\left(z_{01}, a\right)=\{(0,1),(2,1)\}, & H\left(z_{01}, b\right)=\{(1,0),(1,2)\}, \\
H\left(z_{02}, a\right)=\{(1,2),(3,2)\}, & H\left(z_{02}, b\right)=\{(2,1),(2,3)\} .
\end{array}
$$

Перебирая селекторы, получаем:

$$
\begin{aligned}
& h_{1}\left(z_{01}, a\right)=(0,1), \quad h_{1}\left(z_{01}, b\right)=(1,0), \quad h_{2}\left(z_{01}, a\right)=(2,1), \quad h_{2}\left(z_{01}, b\right)=(1,0), \\
& h_{3}\left(z_{01}, a\right)=(0,1), \quad h_{3}\left(z_{01}, b\right)=(1,2), \quad h_{4}\left(z_{01}, a\right)=(2,1), \quad h_{4}\left(z_{01}, b\right)=(1,2) \text {, } \\
& h_{5}\left(z_{02}, a\right)=(1,2), \quad h_{5}\left(z_{02}, b\right)=(2,1), \quad h_{6}\left(z_{02}, a\right)=(3,2), \quad h_{6}\left(z_{02}, b\right)=(2,1) \text {, } \\
& h_{7}\left(z_{02}, a\right)=(1,2), \quad h_{7}\left(z_{02}, b\right)=(2,3), \quad h_{8}\left(z_{02}, a\right)=(3,2), \quad h_{8}\left(z_{02}, b\right)=(2,3) \text {. }
\end{aligned}
$$


Комбинируя любой из первых четырех селекторов с любым из последних четырех, имеем 16 возможных вариантов уравнений (2.1). Далее находим множество $\mathcal{Q}_{1}$ в виде четырех вариантов распределений при условии $z_{01}$ :

$$
\begin{aligned}
& q_{11}\left(z_{1}=(0,1) \mid z_{01}\right)=q_{11}\left(z_{1}=(1,0) \mid z_{01}\right)=0.5, \quad q_{12}\left(z_{1}=(2,1) \mid z_{01}\right)=q_{12}\left(z_{1}=(1,0) \mid z_{01}\right)=0.5, \\
& q_{13}\left(z_{1}=(0,1) \mid z_{01}\right)=q_{13}\left(z_{1}=(1,2) \mid z_{01}\right)=0.5, \quad q_{14}\left(z_{1}=(2,1) \mid z_{01}\right)=q_{14}\left(z_{1}=(1,2) \mid z_{01}\right)=0.5,
\end{aligned}
$$

и еще четырех вариантов распределений при условии $z_{02}$ :

$$
\begin{aligned}
& q_{15}\left(z_{1}=(1,2) \mid z_{02}\right)=q_{15}\left(z_{1}=(2,1) \mid z_{02}\right)=0.5, \quad q_{16}\left(z_{1}=(3,2) \mid z_{02}\right)=q_{16}\left(z_{1}=(2,1) \mid z_{02}\right)=0.5 \\
& q_{17}\left(z_{1}=(1,2) \mid z_{02}\right)=q_{17}\left(z_{1}=(2,3) \mid z_{02}\right)=0.5, \quad q_{18}\left(z_{1}=(3,2) \mid z_{02}\right)=q_{18}\left(z_{1}=(2,3) \mid z_{02}\right)=0.5 .
\end{aligned}
$$

С учетом структуры множества $\mathcal{Q}_{1}$ в множество $\mathcal{D}_{1}$ не может входить никакое другое распределение при условии $z_{01}$, кроме четырех, указанных выше. Аналогичное можно сказать и про распределения при условии $z_{02}$. Итак, $\mathcal{Q}_{1}=\mathcal{D}_{1}$. Множество $\mathcal{M}_{1}$ состоит из распределений при условии $z_{01}$, сосредоточенных на множестве $\{(1,0),(0,1),(2,1),(1,2)\}$ из четырех точек при условиях: $p_{1}+p_{2}+p_{3}+p_{4}=1, p_{i} \leqslant 1 / 2$. Распределения при условии $z_{02}$ в множестве $\mathcal{M}_{1}$ сосредоточены на множестве $\{(2,1),(1,2),(3,2),(2,3)\}$ и удовлетворяют тем же условиям. Рассмотрим, как устроено множество $\mathcal{R}_{1}$ условных распределений для ненаблюдаемого параметра $x_{1}$. Поскольку вероятности на начальном этапе имеют вид $r_{0}\left(x_{0}=y_{0} \mid y_{0}\right)=1$, где $y_{0} \in\{1,2\}$, то и после измерений на первом этапе распределения получают вид $\delta$-функций. Например, $r_{1}\left(x_{1}=0 \mid y_{0}=1, y_{1}=1\right)=1, r_{1}\left(x_{1}=1 \mid y_{0}=1, y_{1}=0\right)=1, r_{1}\left(x_{1}=1 \mid y_{0}=2, y_{1}=2\right)=1$, $r_{1}\left(x_{1}=2 \mid y_{0}=2, y_{1}=1\right)=1$. Рассматриваемый одношаговый процесс нетрудно превратить в многошаговый, если на каждом шаге добавлять независимое вероятностное пространство $\left\{a_{k}, b_{k}\right\}$ с равномерным распределением. При попадании точки на "границу" точка отражается с вероятностью 1 "внутрь" под прямым углом. Во внутренних точках с равной вероятностью происходит переход либо в вертикальное, либо в горизонтальное множество из двух точек. В данном многошаговом процессе последовательность наблюдений $y^{k}=\left[y_{1} ; \ldots ; y_{k}\right]$ позволяет точно определить значение $x_{k}$. Развитие процесса в примере показано на схеме:

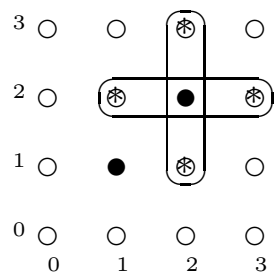

В примере 1 два множества из (3.6) оказались совпадающими. Приведем пример, где все множества в (3.6), а значит и в (3.7), различны.

П р и м е р 2. Рассмотрим снова одношаговую систему с дискретным фазовым пространством $Z_{0}=Z_{1}=\{0,1\}^{2}$. Для удобства занумеруем точки пространства числами $1,2,3,4$, где 1 - это $(1,0)$. Далее нумеруем по часовой стрелке. Вероятностное пространство задается двумя точками $\{a, b\}$ с распределением $P(a)=1 / 3$. Пусть начальное распределение является равномерным. Мультифункция $H$ задается следующим образом: $H(2, a)=\{1,2,3\}, H(2, b)=\{1,2\}$, $H(3, a)=\{2,3,4\}, H(3, b)=\{2,3\}, H(4, a)=\{3,4,1\}, H(4, b)=\{3,4\}, H(1, a)=\{4,1,2\}$, $H(1, b)=\{4,1\}$. При каждом начальном состоянии $i \in 1: 4$ у мультифункции $H$ имеется шесть селекторов. В соответствии с этим множество $\mathcal{Q}_{1}$ при условии 2 состоит из шести распределений

$$
(1,0,0,0), \quad(2 / 3,1 / 3,0,0), \quad(2 / 3,0,1 / 3,0), \quad(1 / 3,2 / 3,0,0), \quad(0,1,0,0), \quad(0,2 / 3,1 / 3,0) .
$$


Здесь $\left(p_{1}, p_{2}, p_{3}, p_{4}\right)$ - набор вероятностей, соответствующих данному состоянию. Аналогично получаем при условиях $3,4,1$ соответственно:

$$
\begin{array}{llllll}
(0,1,0,0), & (0,2 / 3,1 / 3,0), & (0,2 / 3,0,1 / 3), & (0,1 / 3,2 / 3,0), & (0,0,1,0), & (0,0,2 / 3,1 / 3) ; \\
(0,0,1,0), & (0,0,2 / 3,1 / 3), & (1 / 3,0,2 / 3,0), & (0,0,1 / 3,2 / 3), & (0,0,0,1), & (1 / 3,0,0,2 / 3) ; \\
(0,0,0,1), & (1 / 3,0,0,2 / 3), & (0,1 / 3,0,2 / 3), & (2 / 3,0,0,1 / 3), & (1,0,0,0), & (2 / 3,1 / 3,0,0) .
\end{array}
$$

Эти наборы вероятностей выводятся циклической перестановкой. Каждая из них включает состояние с нулевой вероятностью. Теперь заметим, что в множество $\mathcal{D}_{1}$ помимо указанных распределений войдут еще распределения

$$
(1 / 3,1 / 3,1 / 3,0), \quad(0,1 / 3,1 / 3,1 / 3), \quad(1 / 3,0,1 / 3,1 / 3), \quad(1 / 3,1 / 3,0,1 / 3) .
$$

Что касается выпуклых множеств вероятностей, принадлежащих $\mathcal{M}_{1}$ при фиксированном начальном состоянии, то они будут содержать также распределения

$$
(1 / 2,1 / 2,0,0), \quad(0,1 / 2,1 / 2,0), \quad(0,0,1 / 2,1 / 2), \quad(1 / 2,0,0,1 / 2) .
$$

Более того, при условии, например, 2 в $\mathcal{M}_{1}$ войдут все распределения, для которых $p_{1}+p_{2}+p_{3}=$ $1, p_{3} \leqslant 1 / 3$. Рассмотрим построение множества $\mathcal{R}_{1}$ условных распределений. На начальном этапе величины $x_{0}$ и $y_{0}$ являются независимыми. Поэтому условное распределение $x_{0}$ совпадает с безусловным: $P\left(x_{0}=1\right)=P\left(x_{0}=2\right)=0.5$. Измерение $y_{1}$ дает существенную информацию. Так, для третьего распределения при условии 2 будем иметь $q\left(x_{1}=1 \mid 2, y_{1}=\right.$ $0)=1, q\left(x_{1}=0 \mid 2, y_{1}=1\right)=1$. Найдя все условные распределения $q_{m}\left(x_{1}=j \mid i, y_{1}=l\right)$, где $j, l \in 1: 2, i \in 1: 4, m \in 1: 6$, получим множество $\mathcal{R}_{1}$ в виде совокупности распределений $\left\{\sum_{i=1}^{4} q_{m}\left(x_{1}=j \mid i, y_{1}=l\right) / 4 \mid m \in 1: 6\right\}$. Данный процесс можно продолжать, добавляя на каждом шаге независимое вероятностное пространство $\left\{a_{k}, b_{k}\right\}$ с распределением $P\left(a_{k}\right)=1 / 3$ и считая, что мультифункция $H$ постоянна на каждом шаге $k$. Фазовое пространство $Z_{k}=\{0,1\}^{2}$ показано на схеме:

\section{4. Достаточные условия совпадения схем фильтрации для включений}

Нас будут интересовать прежде всего условия совпадения множеств во включениях (3.6). На основное вероятностное пространство налагаются дополнительные условия.

О п р е д е л е н и е 1 . Множество $A \in \mathcal{F}$ с $P(A)>0$ в вероятностном пространстве $(\Omega, \mathcal{F}, P)$ называется атомом, если для всех подмножеств $B$ со свойством $\mathcal{F} \ni B \subset A$ имеем $P(B)=P(A)$ или $P(B)=0$. Вероятностное пространство называется неатомическим, если оно не содержит атомов.

Далее примем

Предположение 1. Основное вероятностное пространство $(\Omega, \mathcal{F}, P)$ является неатомическим.

С учетом [17, лемма 4.9] или [10, теорема 2] получаем теорему.

Теорема 2. Пусть выполнено предположение 1 и все множества $Z_{k}, k \in 1: N$, конечны. Тогда все множества в (3.6), (3.7) совпадают. 
Кратко поясним д о к аз а т е л ь с т в о. Пусть мультифункция $H_{k}: Z_{k-1} \Omega \rightarrow \mathcal{P}\left(Z_{k}\right)$

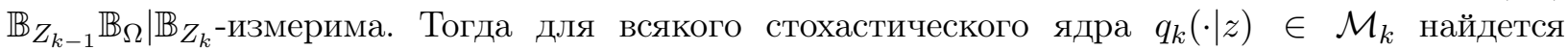
$\mathbb{B}_{Z_{k-1}} \mathbb{B}_{[0,1]} \mid \mathbb{B}_{Z_{k}}$-измеримое отображение $h_{k}$, такое что $q_{k}(D \mid z)=\lambda\left(h_{k}^{-}(D)_{z}\right)$. Здесь $\lambda-$ мера Лебега и множество $\mathcal{M}_{k}$ определяется согласно (3.5). Указанное отображение строится так же, как в [17] или [10], с помощью функции квантили и с учетом конечности множеств $Z_{k}$. Далее используется результат из [10], согласно которому на каждом неатомическом пространстве существует измеримое отображение $\alpha: \Omega \rightarrow[0,1]$ с равномерным распределением. Подставив это отображение в $h_{k}$, получим композицию $h_{k}(z, \alpha(\omega))$, которая обладает нужными свойствами. Итак, $\mathcal{Q}_{k}=\mathcal{D}_{k}=\mathcal{M}_{k}$.

В работе [17] имеется ряд результатов о совпадении множеств $P_{H}=\Delta_{H}=M_{H}$ для мультиотображений из неатомических пространств в интервалы из $\mathbb{R}$, а также утверждается, что возможно перенести результаты на многомерные случайные интервалы. Однако доказательств данного факта в доступной литературе найти не удалось. Контрпримеры также пока не построены, даже в $\mathbb{R}^{2}$. Поэтому рассмотрим более узкие и специальные классы мультифункций и их селекторов.

О п р е д е л е н и е 2. Множество всех борелевских ядер $q_{k}(\cdot \mid z) \in \mathbf{P}_{k}$ на $Z_{k}$ при условии $Z_{k-1}$, для которых существует $\mathbb{B}_{Z_{k-1}} \mathbb{B}_{[0,1]} \mid \mathbb{B}_{Z_{k}}$-измеримое отображение $f_{k}: Z_{k-1}[0,1] \rightarrow Z_{k}$, такое что $\lambda\left(f_{k}^{-}(D)_{z}\right)=q_{k}(D \mid z), \forall D \in \mathbb{B}_{Z_{k}}, \forall z \in Z_{k-1}$, где $\lambda$ - мера Лебега, обозначим через $\tilde{\mathbf{P}}_{k}$ и назовем множеством лебеговских ядер. Пусть $H_{k}$ является $\mathbb{B}_{Z_{k-1}} \mathcal{F} \mid \mathbb{B}_{Z_{k}}$-измеримым мультиотображением в правой части включения (2.9). Измеримый селектор $h_{k} \in S\left(H_{k}\right)$ определим как лебеговский, если $P\left(h_{k}^{-}(D)_{z}\right)=q_{k}(D \mid z), \forall D \in \mathbb{B}_{Z_{k}}, \forall z \in Z_{k-1}$ и $q_{k}(\cdot \mid z) \in \tilde{\mathbf{P}}_{k}$. Множество всех лебеговских селекторов обозначим как $\tilde{S}\left(H_{k}\right)$, а множество всех лебеговских распределений на $Z$ - как $\tilde{\mathbb{P}}(Z)$.

Вопрос о существовании лебеговских селекторов для достаточно широкого класса измеримых мультиотображений остается пока открытым. Назовем мультиотображение простым, если оно имеет конечное число многозначных значений. Рассмотрим множество простых измеримых полуоткрытых прямоугольников в $\mathbb{R}^{2}$, заданных на неатомическом вероятностном пространстве $(\Omega, \mathcal{F}, P)$. Для них множество лебеговских селекторов непусто. Отображение $\Pi(\omega)=(a(\omega), b(\omega)](c(\omega), d(\omega)]$ - измеримый прямоугольник (полуоткрытый), если $a(\omega)<$ $b(\omega), c(\omega)<d(\omega), \forall \omega \in \Omega$, и функции $a, b, c, d$ измеримы. Простые прямоугольники имеют конечное число прямоугольных значений, которые могут пересекаться. Сформулируем лемму.

Лемма 2. Множество $\tilde{S}(\Pi)$ лебеговских селекторов для измеримого простого прямоугольника П на неатомическом полном пространстве непусто.

Д о к а з а т е л ь с т в о. Предположим вначале, что прямоугольник постоянен: $(\omega) \equiv \Pi$. Возьмем непрерывную функцию распределения $F(x, y)$ некоторой двумерной случайной величины со свойствами: неравенство $F(x, y) \leqslant F(u, v)$ влечет $x \leqslant u, y \leqslant v ; q(\Pi)=1$. Здесь $q-$ вероятностная мера на $\mathbb{R}^{2}$, порождаемая функцией $F$. В множестве уровня $\{z \mid F(z) \leqslant \alpha\}$ выберем непрерывный селектор $h(\alpha) \in \mathbb{R}^{2}$, такой что $F(h(\alpha))=\alpha$. Имеем $\lambda(\{\alpha \mid h(\alpha) \leqslant z\})=$ $\lambda(\{\alpha \mid \alpha \leqslant F(z)\})=F(z)$. Таким образом, отображение $h:[0,1] \rightarrow \mathbb{R}^{2}$ обладает функцией распределения $F$. Согласно [17] существует измеримое отображение $\alpha: \Omega \rightarrow[0,1]$ с равномерным распределением, что означает $P(\{\omega \mid \alpha(\omega) \in A\})=\lambda(A)$. Определив композицию $h(\alpha(\omega))$, выводим равенство $P(\{\omega \mid h(\alpha(\omega)) \in \Pi\})=P\left(\left\{\omega \mid \alpha(\omega) \in h^{-}(\Pi)\right\}\right)=\lambda\left(h^{-}(\Pi)\right)=q(\Pi)=1$. Пользуясь полнотой, можем считать, что включение $h(\alpha(\omega)) \in \Pi$ выполняется всюду. Получили искомый селектор из $\tilde{S}(\Pi)$. Одну упомянутую функцию $F$ для прямоугольника можно построить так. Пусть $d=\sqrt{(b-a)^{2}+(d-c)^{2}}$ - длина диагонали. Если $z$ - точка диагонали с координатами $(\bar{x}, \bar{y})$, то на отрезках, соединяющих $z$ с точками $(b, \bar{y})$ и $(\bar{x}, d)$, функция $F(x, y)$ постоянна и равна $\sqrt{(\bar{x}-a)^{2}+(\bar{y}-c)^{2}} / d \in[0,1]$. Функция $F$ удовлетворяет нужным свойствам возрастания и $F(x+\alpha, y+\beta)+F(x, y)-F(x+\alpha, y)-F(x, y+\beta) \geqslant 0$ для всех $\alpha \geqslant 0, \beta \geqslant 0$ и для всех $(x, y) \in \Pi$. Значит, построенная функция действительно является функцией распределения. Рассмотрим измеримый простой прямоугольник. Тогда он может быть представлен в 
виде $\Pi=\bigcup_{i=1}^{n} \Pi_{i} I_{B_{i}}$, где $\left\{B_{1}, \ldots, B_{n}\right\}$ - разбиение $\Omega$ на непересекающиеся множества $B_{i} \in \mathcal{F}$; $I_{B}$ - характеристическая функция множества $B$. Представленная формула означает, что $\Pi \equiv \Pi_{i}$ на $B_{i}$. Определим класс подмножеств $\mathcal{D}=\left\{H_{1} \cap \cdots \cap H_{n} \mid H_{i}=\Pi_{i}\right.$ или $\left.H_{i}=\tilde{\Pi} \backslash \Pi_{i}\right\}=$ $\left\{E_{1}, \ldots, E_{l}\right\}$, где $\tilde{\Pi}-$ наименьший полуоткрытый прямоугольник, содержащий все $\Pi_{i}$. Класс $\mathcal{D}$ является разбиением Пٓ на непересекающиеся подмножества. Каждый элемент $E_{j} \in \mathcal{D}$ в свою очередь подразделяется на полуоткрытые непересекающиеся прямоугольники. Окончательно получим $\bigcup_{i=1}^{n} \Pi_{i}=\bigcup_{j=1}^{m} D_{j}$, где $D_{j}$ - полуоткрытые прямоугольники, причем $D_{i} \cap D_{j}=\varnothing$ и для каждого $D_{j}$ найдется хотя бы одно множество $\Pi_{i}$, такое что $D_{j} \subset \Pi_{i}$. В соответствии с количеством $m$ элементов $D_{j}$ разобьем интервал $(0,1]=\bigcup_{j=1}^{m}\left(a_{j}, b_{j}\right]$ на пересекающиеся части. Выбирая взаимно однозначное отображение из $(0,1]$ на $\left(a_{j}, b_{j}\right]$ и комбинируя их с функциями распределения $F_{j}$ для каждого прямоугольника $D_{j}$, построенными, как и выше, найдем общую функцию $F$ с нужными свойствами. Далее, выбирая селектор $h$ со свойством $F(h(\alpha))=\alpha$ и используя свойство неатомичности, получим нужный селектор из $\Omega$ в $\Pi(\omega)$.

3 а м е ч а н и е. Лемму можно распространить на полуоткрытые прямоугольники в $\mathbb{R}^{n}$. Действительно, в прямоугольнике $\Pi=\prod_{i=1}^{n}\left(a_{i}, b_{i}\right]$ выберем диагональ и обозначим ее длину через $d$. Для любой точки $z$ на диагонали с координатами $\left(\bar{x}_{1}, \ldots, \bar{x}_{n}\right)$ строим $n$ отрезков, соединяющих $z$ с точками $\left(\bar{x}_{1}, \ldots, b_{i}, \ldots, \bar{x}_{n}\right)$. На эти отрезки натягиваем многогранную коническую поверхность и определяем функцию $F$ на этой поверхности постоянной и равной $|z-a| / d$, где $a=\left(a_{1}, \ldots, a_{n}\right)$. Далее рассуждения леммы повторяются.

Важность введенного класса лебеговских селекторов заключается в том, что для простых мультиотображений, заданных на неатомическом пространстве, множества в (3.8) не различаются, если учитывать только селекторы из $\tilde{S}\left(H_{k}\right)$. В случае совпадения множеств в $(3.8)$ для каждого $z$ при конечных множествах $Z_{k}$ совпадают и множества (3.4)-(3.7). Однако для континуальных множеств $Z_{k-1}$ это далеко не очевидно. Назовем мультиотображение $H_{k}$ из правой части включения (2.9) простым, если существуют конечные разбиения $\left\{A_{1}, \ldots, A_{n}\right\}$ и $\left\{B_{1}, \ldots, B_{m}\right\}$ множеств $Z_{k-1}$ и $\Omega$ (соответственно) на непересекающиеся множества, такие что $H_{k}(z, \omega)=\bigcup_{i \in 1: n, j \in 1: m} H_{k}(i, j) I_{A_{i}}(z) I_{B_{j}}(\omega)$, где $H_{k}(i, j) \in \mathbb{B}_{Z_{k}}, A_{i} \in \mathbb{B}_{Z_{k-1}}, B_{j} \in \mathcal{G}_{k}$. Представленная формула означает, что $H_{k}(z, \omega)=H_{k}(i, j)$ на множестве $A_{i} B_{j}$. Назовем борелевское ядро $q(\cdot \mid z)$ простым, если найдется разбиение $\left\{A_{1}, \ldots, A_{l}\right\}$ множества $Z_{k-1}$ такое, что $q(\cdot \mid z) \equiv q_{i}(\cdot)$ на множестве $A_{i}$. Аналогично определяются и простые селекторы из $S\left(H_{k}\right)$ и из $\tilde{S}\left(H_{k}\right)$. Сформулируем теорему об упомянутом совпадении множеств, где меру $P$ на основном вероятностном пространстве будем считать полной.

Теорема 3. Пусть выполнено предположсение 1 и все множества $\tilde{S}\left(H_{k}\right) \neq \varnothing$ для простых мультиотображений $H_{k}$. Тогда множества во включениях (3.6), а значит и в (3.7), совпадают, если учитывать только простые лебеговские селекторы и простые лебеговские ядpa.

Д о к а з а т е л ь с т в о. Его достаточно провести только для первого шага, поскольку для последующих шагов рассуждения аналогичны. Для упрощения опустим индексы $k$, положим $Z=Z_{1}, \mathcal{G}=\mathcal{G}_{1}$ и запишем соотношение $H(z, \omega)=\bigcup_{i \in 1: n, j \in 1: m} H(i, j) I_{A_{i}}(z) I_{B_{j}}(\omega)$, где $H(i, j) \in \mathbb{B}_{Z}, A_{i} \in \mathbb{B}_{Z_{0}}, B_{j} \in \mathcal{G}$. Напомним, что $\sigma$-алгебры $\mathcal{F}_{0}$ и $\mathcal{G}$ независимы, множества $A_{i}$ и $B_{j}$ образуют разбиения множеств $Z_{0}$ и $\Omega$ соответственно. Пусть $n=m=1$, тогда $H(z, \omega) \equiv H$. Возьмем любое ядро $q(\cdot \mid z) \in \tilde{\mathbf{P}}$, для которого $q(D \mid z) \leqslant P^{* z}(D) \quad \forall D \in \mathbb{B}_{Z} \quad \forall z \in Z_{0}$. Но верхняя вероятность $P^{* z}(D)=1$, если $H \cap D \neq \varnothing$. В противном случае $P^{* z}(D)=0$. Значит, $q(H \mid z)=1$. По условию найдется $\mathbb{B}_{Z_{0}} \mathbb{B}_{[0,1]} \mid \mathbb{B}_{Z}$-измеримое отображение $f: Z_{0}[0,1] \rightarrow Z$, такое что $\lambda\left(f^{-}(D)_{z}\right)=q(D \mid z), \forall D \in \mathbb{B}_{Z}, \forall z \in Z_{0}$. Как при доказательстве леммы 2 , существует измеримое отображение $\alpha: \Omega \rightarrow[0,1]$ с равномерным распределением, что означает $P(\{\omega \mid$ $\alpha(\omega) \in A\})=\lambda(A)$. Поскольку $\sigma$-алгебра $\mathcal{G} \subset \mathcal{F}$, то вероятностное пространство $(\Omega, \mathcal{G}, P)$ также является неатомическим. Поэтому функцию $\alpha$ можно считать измеримой относительно $\sigma$ алгебры $\mathcal{G}$. Подставив $\alpha$ в $f$, получим селектор $f(z, \alpha(\omega))$. Для него $P(\{\omega \mid f(z, \alpha(\omega)) \in H\})=$ 
$P\left(\left\{\omega \mid \alpha(\omega) \in f^{-}(H)_{z}\right\}\right)=\lambda\left(f^{-}(H)_{z}\right)=q(H \mid z)=1$. Изменяя, если нужно, функцию $\alpha$ на множестве нулевой меры, получаем включение $f(z, \alpha(\omega)) \in H$ в каждой точке $\omega$. Итак, утверждение доказано для постоянных мультиотображений. Рассмотрим общий случай. Нужно показать, что для любого простого ядра $q(\cdot \mid z) \in \tilde{\mathbf{P}}$, для которого $q(D \mid z) \leqslant P^{* z}(D) \forall D \in \mathbb{B}_{H}$, $\forall z \in Z_{0}$, найдется измеримое отображение $h: Z_{0} \Omega \rightarrow Z$, такое что $P\left(h^{-}(D)_{z}\right)=q(D \mid z)$ $\forall D \in \mathbb{B}_{H}, \forall z \in Z_{0}$. Поскольку ядро является простым, можно полагать, что разбиения постоянства для ядра и мультиотображения совпадают. Иначе берем их измельчение. Более того, можно рассмотреть включения отдельно для каждого множества $A_{i}$ из соответствующего разбиения. Поэтому, не ограничивая общности, полагаем, что $H(\omega)=\bigcup_{j \in 1: m} H(j) I_{B_{j}}(\omega)$, т. е. есть мультиотображение не зависит от $z$, и $q(\cdot \mid z) \equiv q(\cdot) \in \tilde{\mathbb{P}}(Z)$. Пусть $\tilde{H}=\bigcup_{j \in 1: m} H(j)$. Определим класс $\mathcal{D}=\left\{E_{1} \cap \cdots \cap E_{m} \mid E_{j}=H(j)\right.$ или $\left.E_{j}=\tilde{H} \backslash H(j)\right\}=\left\{D_{1}, \ldots, D_{l}\right\}$. Класс $\mathcal{D}$ является разбиением $\tilde{H}$ на непересекающиеся подмножества $D_{i}, i \in 1: l$. Здесь $l<2^{m}$. Далее считается, что пустые множества из $\mathcal{D}$ удалены. Пусть $F: \mathcal{D} \rightarrow\{1, \ldots, l\}-$ биекция такая что $F\left(\bigcup_{i \in I} D_{i}\right)=I$. Рассмотрим мультиотображение $\mathcal{H}=F(H): \Omega \rightarrow \mathcal{P}(\{1, \ldots, l\})$, определенное как $\mathcal{H}(\omega)=F(H(\omega))$. Это будет сильно измеримое мультиотображение из $\Omega$ в конечное множество. Действительно, имеем для $I \subset \mathcal{P}(\{1, \ldots, l\})$ :

$$
\mathcal{H}^{*}(I)=H^{*}\left(\bigcup_{i \in I} D_{i}\right)=\bigcup_{i \in I} \bigcup_{H(j) \supset D_{i}} B_{j} \in \mathcal{G} .
$$

Мы получаем $P_{\mathcal{H}}=M_{\mathcal{H}}$ согласно теореме 2.

Возьмем любое ядро $q(\cdot) \in \tilde{\mathbb{P}}(Z)$, для которого $q(D) \leqslant P_{H}^{*}(D) \forall D \in \mathbb{B}_{\tilde{H}}$. Мы ограничиваемся объединением $\tilde{H}$, поскольку вне этого множества верхняя вероятность равна нулю. Напомним, что $P_{H}^{*}(D)=P\left(H^{*}(D)\right)$. Теперь определим ядро $q^{\prime}(\cdot)$ на $\mathcal{P}(\{1, \ldots, l\})$ следующим образом: $q^{\prime}(I)=q\left(F^{-}(I)\right)$. Мы получаем

$$
\begin{gathered}
q^{\prime}(I)=q\left(F^{-}(I)\right)=q\left(\bigcup_{i \in I} D_{i}\right) \leqslant P_{H}^{*}\left(\bigcup_{i \in I} D_{i}\right)=\sum_{H(j) \cap\left(\bigcup_{i \in I} D_{i}\right) \neq \varnothing} P\left(B_{j}\right) \\
=P\left(\mathcal{H}^{*}(I)\right)=P_{\mathcal{H}}^{*}(I),
\end{gathered}
$$

принимая во внимание (4.1). Поэтому $q^{\prime}(\cdot) \in M_{\mathcal{H}}=P_{\mathcal{H}}$. Из последнего включения выводим существование измеримого селектора $u(\omega) \in \mathcal{H}(\omega) \forall \omega \in \Omega$, такого что $P\left(u^{-}(I)\right)=q^{\prime}(I)$. Введем множества $G_{i}=u^{-}(i) \in \mathcal{G}$ для каждого $i \in 1: l$ и определим мультиотображения

$$
Q_{i}(\omega)= \begin{cases}D_{i}, & \text { если } \omega \in G_{i}, \\ \varnothing, & \text { если } \omega \notin G_{i} .\end{cases}
$$

Рассмотрим ядро $q_{i}(\cdot)$, задаваемое формулой $q_{i}(A)=q\left(A \cap D_{i}\right)$ для всех $A \in \mathbb{B}_{\tilde{H}}$. Тогда $q_{i}(A) \leqslant$ $P\left(Q_{i}^{*}(A)\right) \forall A \in \mathbb{B}_{D_{i}}$, потому что $q_{i}\left(D_{i}\right)=P\left(G_{i}\right)$ для всех $i$ и $P\left(Q_{i}^{*}(A)\right)=P\left(G_{i}\right) \forall A \in \mathbb{B}_{D_{i}}$. Здесь учитываются равенства $P\left(u^{-}(i)\right)=q^{\prime}(i)=q\left(F^{-}(i)\right)$. Для каждого $i$ мы получаем ситуацию с постоянным мультиотображением $Q_{i}$, заданным на неатомическом пространстве $\left(G_{i}, \mathcal{G} \cap G_{i}, P\right)$, где мера $P\left(G_{i}\right) \leqslant 1$, и некоторым ядром $q_{i}(\cdot)$ с $q_{i}\left(D_{i}\right)=P\left(G_{i}\right)=d_{i}$. Вводя новую меру $\tilde{P}=$ $P / d_{i}$, если $d_{i}>0$, сводим задачу к случаю постоянного мультиотображения на вероятностном пространстве, исследованному вначале. Если $d_{i}=0$, номер $i$ исключается из рассмотрения. Значит, для каждого номера $i$ найдется лебеговский селектор $w_{i}: G_{i} \rightarrow D_{i}$ такой, что $P_{w_{i}}(A)=$ $q_{i}(A) \forall A \in \mathbb{B}_{D_{i}}$. Теперь определяем отображение

$$
w: \Omega \rightarrow Z, \text { как } \omega \mapsto w_{i}(\omega), \text { если } \omega \in G_{i} .
$$

Отображение $w$ - измеримый селектор для $H(\omega)$, такой что $P_{w}(D)=q(D), \forall D \in \mathbb{B}_{Z}$.

Проверим необходимые свойства отображения $w$. В самом деле, $\left\{G_{1}, \ldots, G_{l}\right\}=\left\{u^{-}(1), \ldots\right.$, $\left.u^{-}(l)\right\}$ - это разбиение множества $\Omega$. Кроме того, $Q_{i}(\omega) \neq \varnothing \forall \omega \in G_{i}$. Значит, отображение $w$ 
корректно определено. Если $\omega \in G_{i}$, то

$$
\begin{gathered}
w(\omega)=w_{i}(\omega) \in Q_{i}(\omega)=D_{i}=F^{-}(i)=F^{-}(u(\omega)) \in F^{-}(\mathcal{H}(\omega)) \\
=\left\{D_{j} \mid D_{j} \subset H(\omega)\right\}, \quad \text { следовательно } w(\omega) \in H(\omega) .
\end{gathered}
$$

Для множества $A \in \mathbb{B}_{Z}$ имеем $w^{-}(A)=\bigcup_{i \in 1: l}\left(w_{i}^{-}(A) \cap G_{i}\right) \in \mathcal{G}$. Итак, $w$ - измеримый лебеговский селектор для $H$. Пусть опять $A \in \mathbb{B}_{Z}$, тогда

$$
\begin{gathered}
P_{w}(A)=\sum_{i \in 1: l} P\left(w^{-}(A) \cap G_{i}\right)=\sum_{i \in 1: l} P\left(w_{i}^{-}(A) \cap G_{i}\right)=\sum_{i \in 1: l} q_{i}(A) \\
=\sum_{i \in 1: l} q\left(A \cap D_{i}\right)=q(A) .
\end{gathered}
$$

Следовательно, утверждение теоремы доказано.

\section{Заключение}

- Рассмотрены три способа оценивания состояний для многошаговых стохастических включений, основанные на разных способах формирования множества переходных вероятностей.

- Показано на примерах, что эти способы могут приводить к различным множествам условных распределений для текущих ненаблюдаемых состояний процесса.

- Поставлен вопрос о достаточных условиях совпадения рассмотренных схем фильтрации для включений и доказано, что для конечных фазовых пространств эти схемы совпадают в случае неатомического вероятностного пространства.

- Введен новый класс лебеговских селекторов для произвольных мультиотображений и показано, что он не пуст, в частности, для измеримых простых прямоугольников на неатомическом вероятностном пространстве.

- Доказано, что в лебеговском классе для простых включений и селекторов, заданных на неатомическом вероятностном пространстве, схемы фильтрации также совпадают.

\section{СПИСОК ЛИТЕРАТУРЫ}

1. Ananyev B.I. One problem of statistically uncertain estimation // Proc. of Intern. Conf. SCDG2019: Short paper (Yekaterinburg, 16-20 Sept. 2019) / IMM UB of RAS. Yekaterinburg, 2019. P. 375-379. ISBN 978-5-8295-0652-0 .

2. Ананьев Б.И., Аникин С.А. Задача восстановления входных воздействий при коммуникационных ограничениях // Автоматика и телемеханика. 2009. № 7. С. 73-84.

3. Ананьев Б.И. О коррекции движения при коммуникационных ограничениях // Автоматика и телемеханика. 2010. № 3. С. 3-15.

4. Кац И.Я., Куржанский А.Б. Минимаксное оценивание в многошаговых системах // Докл. АН CCCP. 1975. Т. 221, № 3. С. 535-538.

5. Anan'ev B.I. Minimax estimation of statistically uncertain systems under the choice of a feedback parameter // J. Math. Systems, Estimation, and Control. 1995. Vol. 5, no. 2. P. 1-17.

6. Лебедев М.В., Семенихин К.В. Минимаксная фильтрация в стохастической дифференциальной системе с нестационарными возмущениями неизвестной интенсивности // Изв. РАН. Теория и системы управления. 2007. № 2. С. 45-56.

7. Nguyen Hung T. et al. Computing statistics under interval and fuzzy uncertainty. Berlin; Heidelberg: Springer-Verlag, 2012. 432 p. doi: 10.1007/978-3-642-24905-1.

8. Kisielewicz M. Relationship between attainable sets of functional and set-valued functional stochastic inclusions. Stochastic Anal. Appl. 2016. Vol. 34, no. 6. P. 1094-1110.

9. Raol J.R., Gopalratnam G., Twala B. Nonlinear filtering. Concepts and engineering applications. Boca Raton: CRC Press, Taylor \& Francis Group, 2017. 556 p. 
10. Miranda E., Couso I., Gil P. Approximations of upper and lower probabilities by measurable selections // Information Sciences. 2010. Vol. 180, no. 8. P. 1407-1417. doi: 10.1016/j.ins.2009.12.005 .

11. Бертсекас Д., Шрив С. Стохастическое оптимальное управление. М.: Наука, 1985. 279 с.

12. Ширяев А.Н. Вероятность-1: в 2 кн. Кн. 1. М.: МЦНМО, 2004. 520 с.

13. Неве Ж. Математические основы теории вероятностей. М.: Мир, 1969. 431 с.

14. Борисович Ю.Г. и др. Введение в теорию многозначных отображений и дифференциальных включений. М.: КомКнига, 2005. 215 с.

15. Nguyen Hung T. On random sets and belief functions // J. Math. Anal. Appl. 1978. Vol. 65, no. 3. P. 531-542. doi: 10.1016/0022-247X(78)90161-0 .

16. Himmelberg C. Measurable relations // Fund. Math. 1975. Vol. 87. P. 53-72. doi: $10.4064 / \mathrm{fm}-87-1-53-72$.

17. Miranda E., Couso I., Gil P. Random intervals as a model for imprecise information // Fuzzy Sets and Systems. 2005. Vol. 154. P. 386-412. doi: 10.1016/j.fss.2005.03.001.

Поступила 13.11.2019

После доработки 22.01.2020

Принята к публикации 27.01.2020

Ананьев Борис Иванович

д-р физ.-мат. наук

ведущий научный сотрудник

Институт математики и механики им. Н. Н. Красовского УрО РАН

г. Екатеринбург

e-mail: abi@imm.uran.ru

\section{REFERENCES}

1. Ananyev B.I. One problem of statistically uncertain estimation. In: Proc. Intern. Conf. SCDG2019 (Yekaterinburg, 16-20 Sept.), 2019, Yekaterinburg: IMM UB of RAS, pp. 375-379.

ISBN: 978-5-8295-0652-0 .

2. Anan'ev B.I., Anikin S.A. Problem of reconstructing input signals under communication constraints. Autom. Remote Control, 2009, vol. 70, no. 7, pp. 1153-1164. doi: 10.1134/S0005117909070078.

3. Anan'ev B.I. Correction of motion under communication constraints. Autom. Remote Control, 2010, vol. 71, no. 3, pp. 367-378. doi: 10.1134/S000511791003001X.

4. Kats I.Ya., Kurzhanski A.B. Minimax estimation in multistage systems. Dokl. Akad. Nauk SSSR, 1975, vol. 221, no. 3, pp. 535-538 (in Russian).

5. Anan'ev B.I. Minimax estimation of statistically uncertain systems under the choice of a feedback parameter. J. Math. Systems, Estimation, and Control, 1995, vol. 5, no. 2, pp. 1-17.

6. Lebedev M.V., Semenikhin K.V. Minimax filtering in a stochastic differential system with non-stationary perturbations of unknown intensity. J. Comput. Syst. Sci. Int.., 2007, vol. 46, no. 2, pp. 206-217. doi: $10.1134 /$ S1064230707020062.

7. Nguyen Hung T. et al. Computing statistics under interval and fuzzy uncertainty. Berlin; Heidelberg: Springer-Verlag, 2012. 432 p. doi: 10.1007/978-3-642-24905-1.

8. Kisielewicz M. Relationship between attainable sets of functional and set-valued functional stochastic inclusions. Stochastic Analysis and Applications, 2016, vol. 34, no. 6, pp. 1094-1110.

9. Raol J.R., Gopalratnam G., Twala B. Nonlinear filtering. Concepts and engineering applications. Boca Raton: CRC Press, Taylor \& Francis Group, 2017. ISBN: 9781498745178.

10. Miranda E., Couso I., Gil P. Approximations of upper and lower probabilities by measurable selections. Information Sciences, 2010, vol. 180, no. 8, pp. 1407-1417. doi: 10.1016/j.ins.2009.12.005 .

11. Bertsekas D.P., Shreve S.E. Stochastic optimal control: the discrete time case. Burlington, MA: Elsevier, 1978, 341 p. ISBN: 9780080956480 . Translated to Russian under the title Stokhasticheskoe optimal'noe upravlenie: sluchai diskretnogo vremeni. Moscow: Nauka Publ., 1985, 279 p.

12. Shiryaev A.N. Probability. N Y etc.: Springer-Verlag, 1995, Ser. Graduate Texts in Mathematics, vol. 95, 624 p. ISBN: 0387945490 . Original Russian text (3rd ed.) published in Shiryaev A.N. Veroyatnost'-1. Moscow: Nauka Publ., 2004, 520 p. 
13. Neveu J. Mathematical foundations of the calculus of probability. San Francisco: Holden-Day, 1965,223 p. Translated to Russian under the title Matematicheskie osnovy teorii veroyatnostei. Moscow: Mir Publ., 1969, 309 p.

14. Borisovich Yu.G., Gel'man B.D., Myshkis A.D., Obukhovskii V.V. Vvedenie v teoriyu mnogoznachnykh otobrazhenii $i$ differentsial'nykh vklyuchenii [Introduction to the theory of multivalued maps and differential inclusions]. Moscow: KomKniga Publ., 2005, 215 p. ISBN: 5-484-00172-2 .

15. Nguyen Hung T. On random sets and belief functions. J. Math. Anal. Appl., 1978, vol. 65, no. 3, pp. 531-542. doi: 10.1016/0022-247X(78)90161-0 .

16. Himmelberg C. Measurable relations. Fund. Math., 1975, vol. 87, pp. 53-72. doi: 10.4064/fm-87-1-53-72 .

17. Miranda E., Couso I., Gil P. Random intervals as a model for imprecise information. Fuzzy Sets and Systems, 2005, vol. 154, no. 3, pp. 386-412. doi: 10.1016/j.fss.2005.03.001.

Received November 13, 2019

Revised January 22, 2020

Accepted January 27, 2020

Boris Ivanovich Ananyev, Dr. Phys.-Math. Sci., Krasovskii Institute of Mathematics and Mechanics of the Ural Branch of the Russian Academy of Sciences, Yekaterinburg, 620108 Russia, e-mail: abi@imm.uran.ru .

Cite this article as: B. I. Ananyev. Estimation of states of multistage stochastic inclusions, Trudy Instituta Matematiki i Mekhaniki URO RAN, 2020, vol. 26, no. 1, pp. 12-26. 